Article

\title{
A Tunable CW Orange Laser Based on a Cascaded MgO:PPLN Single-Pass Sum-Frequency Generation Module
}

\author{
Dismas K. Choge ${ }^{1,2}{ }^{10}$, Huai-Xi Chen ${ }^{1}$, Bao-Lu Tian ${ }^{1}$, Yi-Bin Xu ${ }^{1}$, Guang-Wei Li ${ }^{1}$ \\ and Wan-Guo Liang ${ }^{1, *}$ \\ 1 Fujian Institute of Research on the Structure of Matter, Chinese Academy of Sciences, Fuzhou 350002, China; \\ dchoge@uoeld.ac.ke (D.K.C.); hxchen@fjirsm.ac.cn (H.-X.C.); tianbaolu1992@163.com (B.-L.T.); \\ xuyibin@fjirsm.ac.cn (Y.-B.X.); gwli@fjirsm.ac.cn (G.-W.L.) \\ 2 University of Chinese Academy of Sciences, Beijing 100049, China \\ * Correspondence: wgl@fjirsm.ac.cn; Tel.: +86-0591-8371-8125
}

Received: 27 January 2018; Accepted: 8 March 2018; Published: 15 March 2018

\begin{abstract}
We report an all-solid-state continuous wave (CW) tunable orange laser based on cascaded single-pass sum-frequency generation with fundamental wavelengths at 1545.7 and $975.2 \mathrm{~nm}$ using two quasi-phase-matched (QPM) MgO-doped periodically poled lithium niobate (MgO:PPLN) crystals. Up to $10 \mathrm{~mW}$ of orange laser is generated in the cascaded module corresponding to a $10.4 \% / \mathrm{W}$ nonlinear conversion efficiency. The orange output showed a temperature tuning rate of $\sim 0.05 \mathrm{~nm} /{ }^{\circ} \mathrm{C}$, and the beam quality $\left(\mathrm{M}^{2}\right)$ value of the orange laser was about 2.0. We use this technique to combine the high efficiency offered by uniformly poled crystals and the broad input wavelength acceptance characteristic of step-chirped structures.
\end{abstract}

Keywords: lithium niobate; sum frequency generation; quasi phase-matching; lasers

\section{Introduction}

Orange lasers with wavelengths near $\sim 600 \mathrm{~nm}$ play an important role in a number of scientific applications such as photodynamic therapy [1], flow cytometry for excitation of fluorescent probes of $595 \mathrm{~nm}$ excitation wavelength [2], spectroscopy [3], astronomy [4], optogenetics and neuroscience [5], and laser projection displays [6]. So far, however, there have been no available conventional diode lasers in this spectral region due to a lack of suitable direct band gap materials [7]. Therefore, compact, stable, and efficient all-solid-state laser sources generated by nonlinear effects have gained popularity over the past thirty years with a view of overcoming this limitation. The abilities of sum frequency generation (SFG) to efficiently mix two fundamental wavelengths to generate a third wavelength is a potential technique to attain such new wavelengths with periodically poled ferroelectric crystals [8]. On the other hand, the quasi phase-matching (QPM) technique using periodically poled lithium niobate (PPLN) is quite attractive for implementing broadband SFG owing to its wide optical transparency window (350-5000 $\mathrm{nm})$, a large nonlinear coefficient $\left(d_{33}\right)$, a flexible design, and a high conversion efficiency [9]. However, reaching high efficiencies in broadband wavelength conversion remains a challenge. Nevertheless, in order to increase the input wavelength acceptance range and achieve the high efficiency of a QPM process, complementary properties of uniform poled and step-chirped crystals in a cascaded configuration can be exploited as proposed herein. The pump and signal lasers are focused into the first crystal and the generated sum frequency light co-propagates in the second crystal in which the pump and the signal are re-focused. In both crystals, SFG occurs separately, increasing the total output power and, consequently, improving the overall conversion efficiency.

Previous studies demonstrating the generation of continuous wave $(\mathrm{CW})$ coherent light using cascaded crystals have been reported. For instance, a two-crystal cascade to generate $491 \mathrm{~nm}$ and 
$429 \mathrm{~nm}$ light with conversion efficiencies of $2.4 \% / \mathrm{W}$ and $3.1 \% / \mathrm{W}$ respectively in $\mathrm{KNbO}_{3}$ crystals was demonstrated [10]. Up to $13 \mathrm{~W}$ of green light at $532 \mathrm{~nm}$ with $5 \% / \mathrm{W}$ conversion efficiency based on two-crystal cascade PPLT crystals has also been reported [11]. Using two PPLN crystals, $780 \mathrm{~nm}$ second harmonic generation has been achieved with $5.6 \% / \mathrm{W}$ conversion efficiency. Recently, $5.5 \mathrm{~W}$ SFG of green light was demonstrated in a two-crystal cascade of PPLN and PPLT crystals with a 50\% conversion efficiency of the total fundamental power [12]. In the orange spectral region, a tunable orange laser from $601 \mathrm{~nm}$ to $604 \mathrm{~nm}$ range was successfully generated by tuning the temperature of quasi-periodically poled lithium tantalate (LT) superlattice from 150 to $195^{\circ} \mathrm{C}$, yielding a maximum output of $310 \mathrm{~mW}$ [13]. Up to $23 \mathrm{~mW}$ of narrow-line $593 \mathrm{~nm}$ light via intracavity SFG of a CW dual-wavelength $\mathrm{Nd}: \mathrm{YVO}_{4}$ laser has also been demonstrated using two volume Bragg gratings in a three-mirror cavity configuration with a temperature tunability of $\sim 5 \mathrm{pm} /{ }^{\circ} \mathrm{C}$ [14]. Recently, also, more than $1 \mathrm{~W}$ all-solid state optical parametric oscillator (OPO) in the spectral range from 605 to $616 \mathrm{~nm}$ based on MgO:PPLN with different poling sections was reported [15]. In this scheme, tuning was achieved by varying the crystal position, temperature and etalon tilt angle. Whilst various schemes of generating visible light in the $600 \mathrm{~nm}$ spectral range have been extensively studied, no work has been reported on high efficiency, tunable wavelength conversion by cascaded nonlinear crystals in this range.

Here, we report the generation of all-solid state, efficient, tunable orange light by single-pass sum frequency mixing of $\sim 1545.7 \mathrm{~nm}$ and $\sim 975.2 \mathrm{~nm}$ laser sources in two cascaded MgO:PPLN bulk crystals. The proposed configuration offers the advantage of combining desirable solid-state properties such as the tunability of pump lasers in the $1527-1565 \mathrm{~nm}$ range, the high output power of $\sim 980 \mathrm{~nm}$ signal laser module, high conversion efficiency offered by uniformly poled QPM crystal and wide input wavelength acceptance range due to a step-chirped (SC-PPLN) crystal. If, for instance, a broadband laser source is used rather than a narrow band highly coherent source for wavelength mixing, multiple wavelengths can be generated simultaneously in SC-PPLN. Up to 7.1 $\mathrm{mW}$ SFG output power was detected for the $50 \mathrm{~mm}$ PPLN crystal, $4.3 \mathrm{~mW}$ for the $20 \mathrm{~mm}$ SC-PPLN crystal, and $10 \mathrm{~mW}$ for the cascaded module corresponding to $7.4 \% / \mathrm{W}, 4.5 \% / \mathrm{W}$, and $10.4 \% / \mathrm{W}$ nonlinear conversion efficiencies of the total input power of the fundamental lasers, respectively. To the best of our knowledge, no work has been reported on the generation of an orange laser using MgO:PPLN bulk crystal in terms of cascaded single-pass sum frequency mixing of uniformly poled and SC-PPLN crystals, which has the advantage of tunability and high conversion efficiency.

\section{Theoretical Considerations}

For two fundamental lasers with input powers $P_{1}$ and $P_{2}$ incident on a nonlinear crystal of length $L$, the theoretical sum frequency output power can be estimated using the relation

$$
P_{S F G}=\frac{32 \pi^{2} d_{e f f}^{2} P_{1} P_{2} L}{n_{S F G} \varepsilon_{0} \lambda_{S F G}^{2} c\left(n_{1} \lambda_{1}+n_{2} \lambda_{2}\right)}
$$

and the corresponding conversion efficiency can be given as

$$
\eta=\frac{P_{S F G}}{P_{1} P_{2}}
$$

where $c$ is the speed of light in vacuum, $\varepsilon_{0}$ is the permittivity in vacuum, and $d_{\text {eff }}=2 d_{33} / \pi$ $\left(d_{33}=27.2 \mathrm{pm} / \mathrm{V}\right)$ is the effective nonlinear coefficient of MgO:PPLN [16]. $n_{S F G}, n_{1}$, and $n_{2}$ are refractive indices at $\lambda_{S F G}, \lambda_{1}$, and $\lambda_{2}$, respectively. For the cascaded module, the expected conversion efficiency for two crystals can be shown as

$$
\eta_{C 1+C 2}=\left(\sqrt{\eta_{C 1}}+\sqrt{\eta_{C 2}}\right)^{2}
$$


where $\eta_{C 1}$ and $\eta_{C 2}$ are the nonlinear efficiencies measured individually for Crystals 1 and 2, respectively, which follows from the superposition principle as detailed in [17].

\section{Experimental Setup}

The setup used in our experiment for cascaded MgO:PPLN single-pass sum-frequency generation is shown in Figure 1. The output of wavelength division multiplexer (WDM) tunable laser source (TLS) with a linewidth of $<100 \mathrm{kHz}$ (FWHM) in the C-band (1527-1565 nm) is amplified using an erbium-doped fiber amplifier (EDFA). It is combined with a $\sim 980 \mathrm{~nm}$ module diode laser (RMS centre wavelength $973.9 \mathrm{~nm}$ and $0.20 \mathrm{~nm}$ spectral width) by a $980 / 1550 \mathrm{~nm}$ wavelength division multiplexer (WDM) with 0.26 and $0.28 \mathrm{~dB}$ insertion losses at $980 \mathrm{~nm}$ and $1550 \mathrm{~nm}$ respectively. The output from the single mode fiber (SMF) that is terminated with a collimator is passed through the first nonlinear uniformly poled MgO:PPLN crystal. The two overlapped input beams are focused into the first crystal and the SFG of the first crystal together with the input beams are re-focused into the second SC-PPLN nonlinear crystal with a $125 \mathrm{~mm}$ focal length plano-convex lenses.

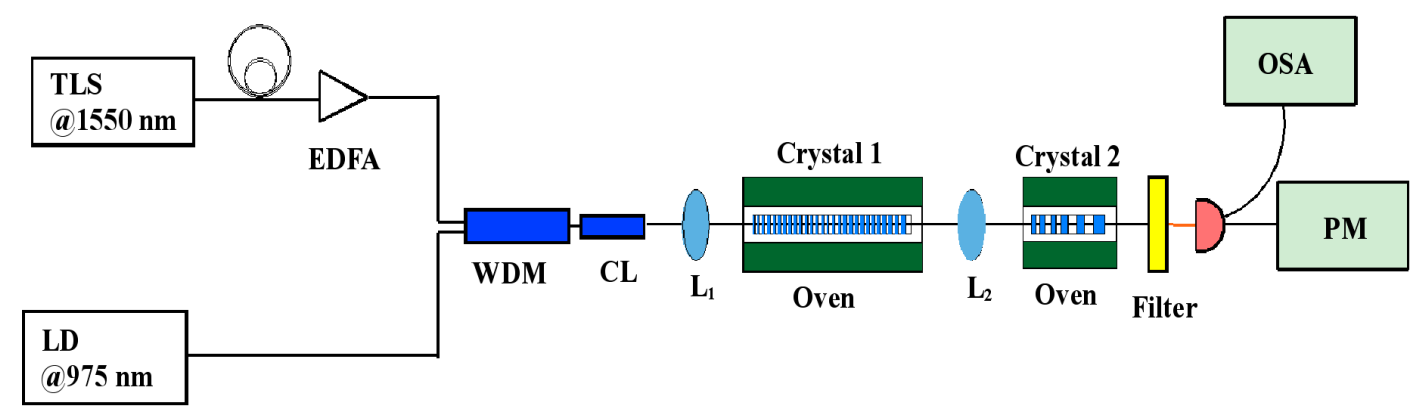

Figure 1. Schematic of the experimental setup. The beams are focused into single-pass cascaded module to generate orange output by sum frequency generation (SFG). EDFA: erbium-doped fiber amplifier; WDM: wave division multiplexer; $\mathrm{CL}$ : collimator; $\mathrm{L}_{1}$ and $\mathrm{L}_{2}$ : lenses; PM: power meter; OSA: optical spectrum analyzer; TLS, tunable laser source; LD, laser diode.

Here, the period of the first MgO:PPLN crystal is $10.3 \mu \mathrm{m}$ and the length is $50 \mathrm{~mm}$. The second MgO:PPLN crystal is a step-chirped structure with poling periods from 10.1 to $10.5 \mu \mathrm{m}$ with a precision of $0.1 \mu \mathrm{m}$ increase and the length is $20 \mathrm{~mm}$ with five equal sections. The SFG phase-matching wavelength for Crystal 1 is $1544.9 \mathrm{~nm}$, while Crystal 2 showed two phase matching wavelengths at $1545.7 \mathrm{~nm}$ and $1556.6 \mathrm{~nm}$. Both crystals were temperature-stabilized close to $31^{\circ} \mathrm{C}$ and $25^{\circ} \mathrm{C}$ for QPM, respectively. The output power and spectra of the up-converted orange output beam after an optical filter was measured using a laser power meter (PM100D, Thorlabs, Newton, NJ, USA) and a fiber spectrometer (BIM-6001, Brolight, Hangzhou, China) with a resolution of $0.35-1 \mathrm{~nm}$, respectively, whereas the $\mathrm{M}^{2}$ factor was measured using knife-edge method with Beamgage (Ophir-Spiricon, Inc., Jerusalem, Israel) laser beam analyzer.

\section{Results and Discussion}

\subsection{Power Dependence}

The maximum input fundamental powers were measured before Crystal 1 to be $418 \mathrm{~mW}\left(P_{1}\right)$ at $1545.7 \mathrm{~nm}\left(\lambda_{1}\right)$ and $229 \mathrm{~mW}\left(P_{2}\right)$ at $975.2 \mathrm{~nm}\left(\lambda_{2}\right)$. We estimated the beam waist radii of $\lambda_{1}$ and $\lambda_{2}$ to be $\sim 68 \mu \mathrm{m}$ and $\sim 43 \mu \mathrm{m}$ inside the crystals, respectively. A comparison of the resulting maximum output powers and corresponding conversion efficiencies with expected calculated values are summarized in Table 1. Perhaps, the total orange output power can be enhanced by anti-reflection (AR) coating the end faces of each crystal in order to minimize Fresnel reflection losses. We can infer from Table 1 that, in comparison to single crystals, the conversion efficiency is higher in the cascaded module 
although lower than the expected conversion efficiency. We attribute the losses to the relative phase offset between the fundamental and SFG beams primarily due to dispersion in air and focusing lenses. The refractive nature of the lens causes color aberration since the focusing lengths for both fundamental wavelengths are different and consequently different beam waist radii results in the second crystal. In order to optimize the conversion efficiency, these limitations can be overcome by focusing the fundamental sources separately into the second crystal. Other possible reasons for the discrepancy between experimental and calculated values may originate from uneven temperature distribution within the crystals and/or poling imperfections in the QPM structure.

Table 1. Calculated and experimental comparison of output powers and conversion efficiencies in single crystals and cascaded module.

\begin{tabular}{|c|c|c|c|c|}
\hline \multirow{2}{*}{ Crystal } & \multicolumn{2}{|c|}{$P_{\text {SFG }}(\mathrm{mW})$} & \multicolumn{2}{|c|}{ Conversion Efficiency $\left(\% \mathrm{~W}^{-1}\right)$} \\
\hline & Calculated $^{a}$ & Experiment & Calculated $^{\mathrm{b}}$ & Experiment \\
\hline Crystal 1 & 38.2 & 7.1 & 39.9 & 7.4 \\
\hline Crystal 2 & 15.2 & 4.3 & 15.9 & 4.5 \\
\hline Cascaded module & - & 10.0 & 23.4 & 10.4 \\
\hline
\end{tabular}

Figure 2a shows the $\mathrm{CW}$ orange power as a function of the total input power incident on the two individual crystals and for the cascaded case. The TLS power, $P_{1}$ was fixed at $418 \mathrm{~mW}$ (maximum) and the LD power, $P_{2}$ was increased steadily to a maximum value of $229 \mathrm{~mW}$ by varying the operating current. Figure $2 \mathrm{~b}$ shows the measured beam profiles when $P_{1}$ and $P_{2}$ were $418 \mathrm{~mW}$ and $229 \mathrm{~mW}$, respectively. For all the cases, the SFG beam was nearly identical in the horizontal direction with $M_{x}^{2} \sim 2.1$ at $595.9 \mathrm{~nm}, 597.3 \mathrm{~nm}$, and $596.9 \mathrm{~nm}$ for Crystal 1, Crystal 2, and the cascaded module, respectively. In the vertical direction, we measured $M_{y}^{2} \sim 1.9, M_{y}^{2} \sim 2.0$, and $M_{y}^{2} \sim 4.1$ for Crystal 1, Crystal 2, and the cascaded module, respectively.

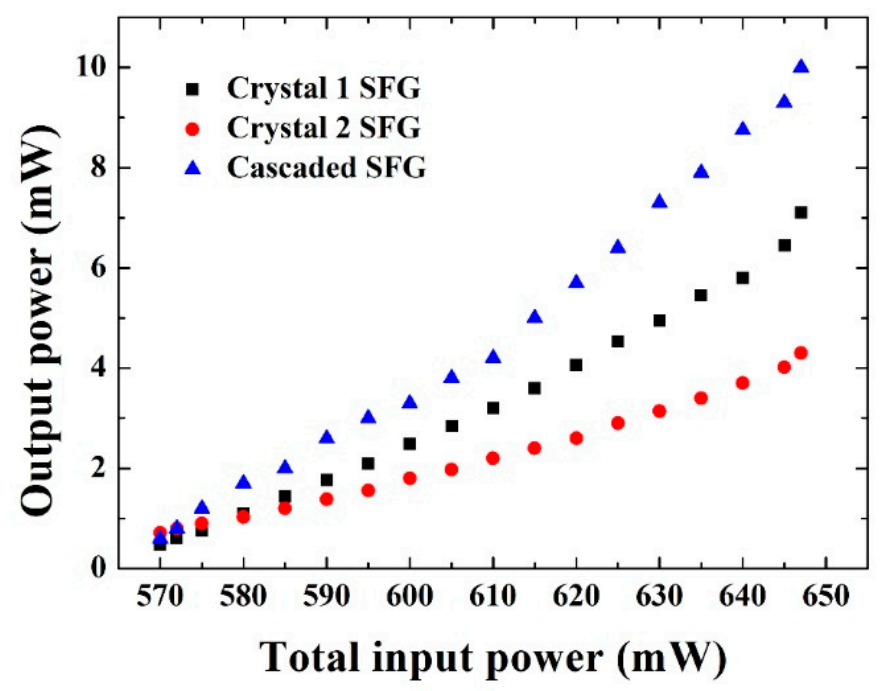

(a)

Figure 2. Cont. 
Crystal 1

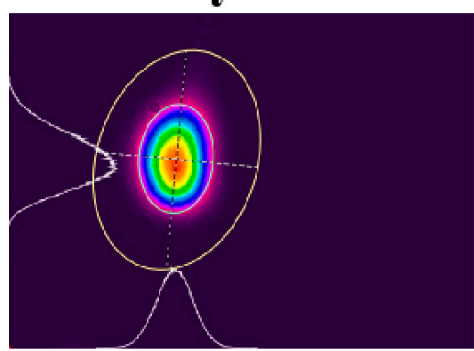

$M^{2} \sim 2.1, M^{2} \sim 1.9$
Crystal 2

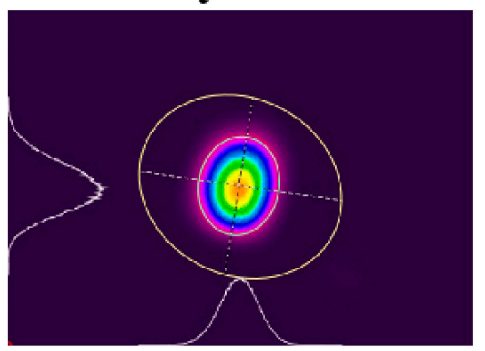

$M^{2} \sim 2.1, M^{2} \sim 2.0$
Cascaded

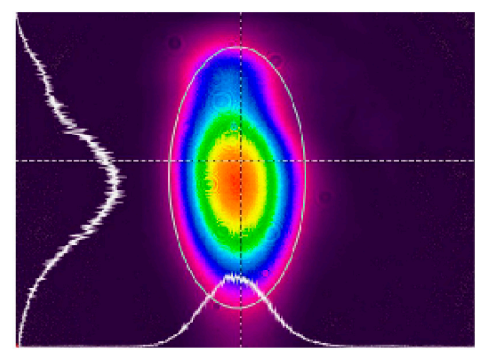

$M^{2} \sim 2.1, M^{2} \sim 4.1$

(b)

Figure 2. (a) Power of the generated orange light as a function of the total input fundamental power. (b) Beam profiles at $595.9 \mathrm{~nm}, 597.3 \mathrm{~nm}$, and $596.9 \mathrm{~nm}$ for the three cases studied taken when $P_{1}$ and $P_{2}$ were $418 \mathrm{~mW}$ and $229 \mathrm{~mW}$, respectively.

\subsection{Output Spectra, Tunability, and Beam Stability}

Figure 3 shows the normalized experimental spectra for the SFG process in Crystal 1, Crystal 2, and the cascaded module. The intensities are normalized to the maximum detection limit of our spectrometer.

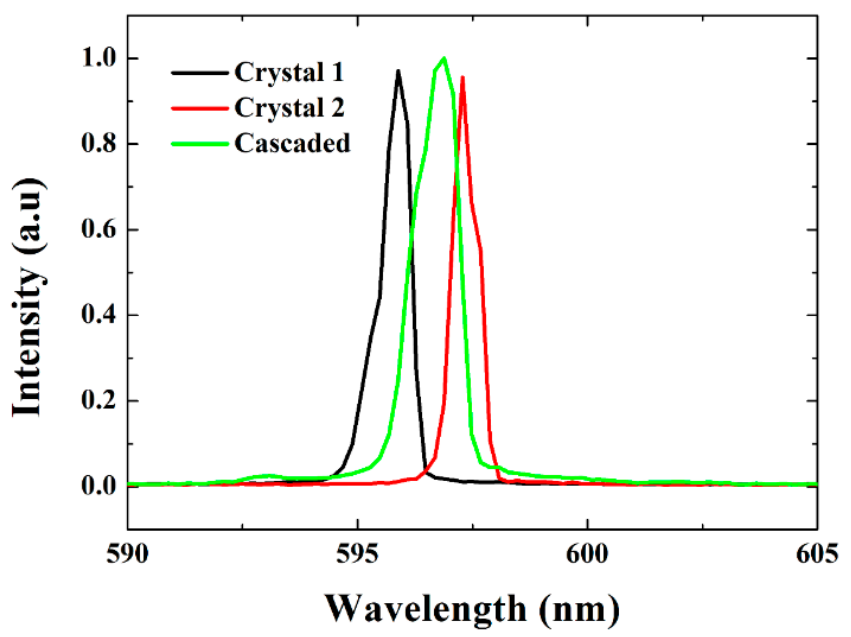

Figure 3. Normalized spectrum of the generated orange light for Crystal 1 (black), Crystal 2 (red), and the cascaded module (green).

We further established the tunability of the orange output by increasing the temperature of Crystal 1 from 25 to $90{ }^{\circ} \mathrm{C}$, while simultaneously tuning the pump laser from 1527 to $1565 \mathrm{~nm}$ (upper horizontal axis) as seen in Figure 4. The corresponding output wavelengths are shown in the lower horizontal axis with a spectral resolution of $\sim 0.75 \mathrm{~nm}$. As the input pump wavelength was tuned, the SFG intensity exhibited oscillations as seen in Figure 5. While Crystal 1 showed a single phase matching point at $1544.9 \mathrm{~nm}$, Crystal 2 revealed two phase matching points at $1545.7 \mathrm{~nm}$ and $1556.5 \mathrm{~nm}$. The cascaded module has a broader input wavelength acceptance range compared to Crystals 1 and 2 . This technique allows the pump wavelengths ranging from 1527 to $1545 \mathrm{~nm}$ to be utilized for SFG, which is not achievable with individual crystals. It is worth noting that the orange intensity dependence on the pump wavelength shown in Figure 5 (green curve) for the cascaded case does not fit to Equation (3) as expected, from which we can conclude that the theory is not valid for this case. 


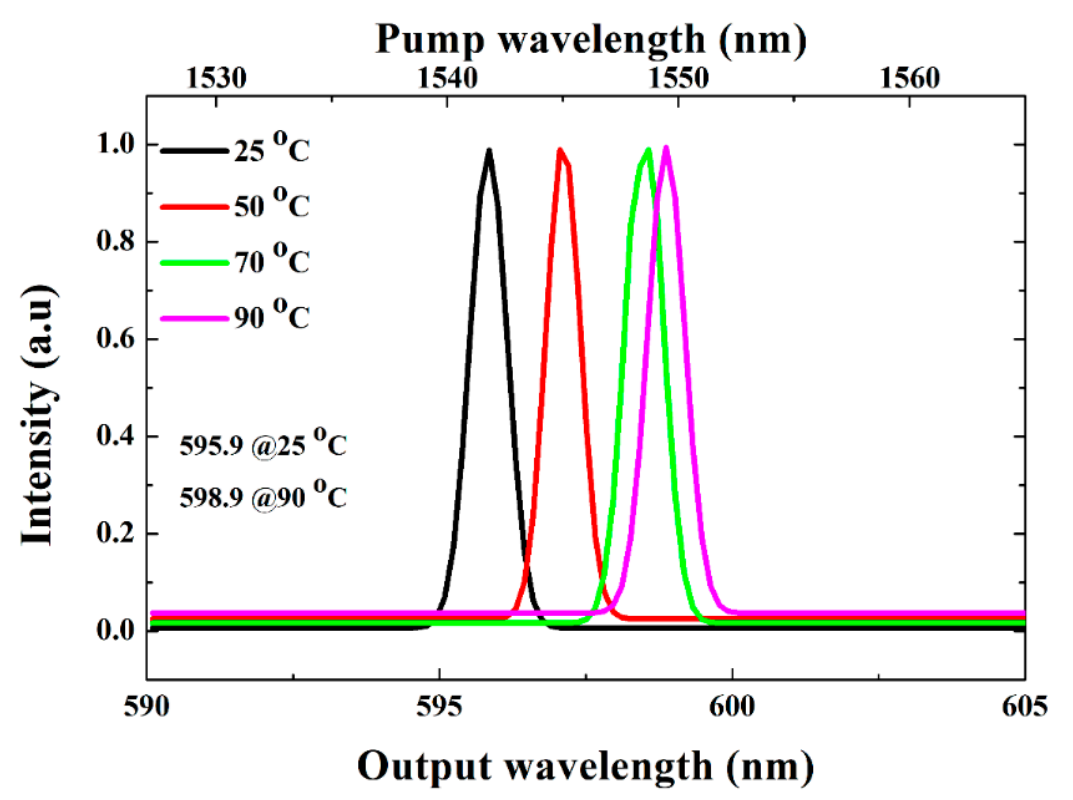

Figure 4. Spectra of orange light tuned across the 595.9-598.9 nm wavelength range.

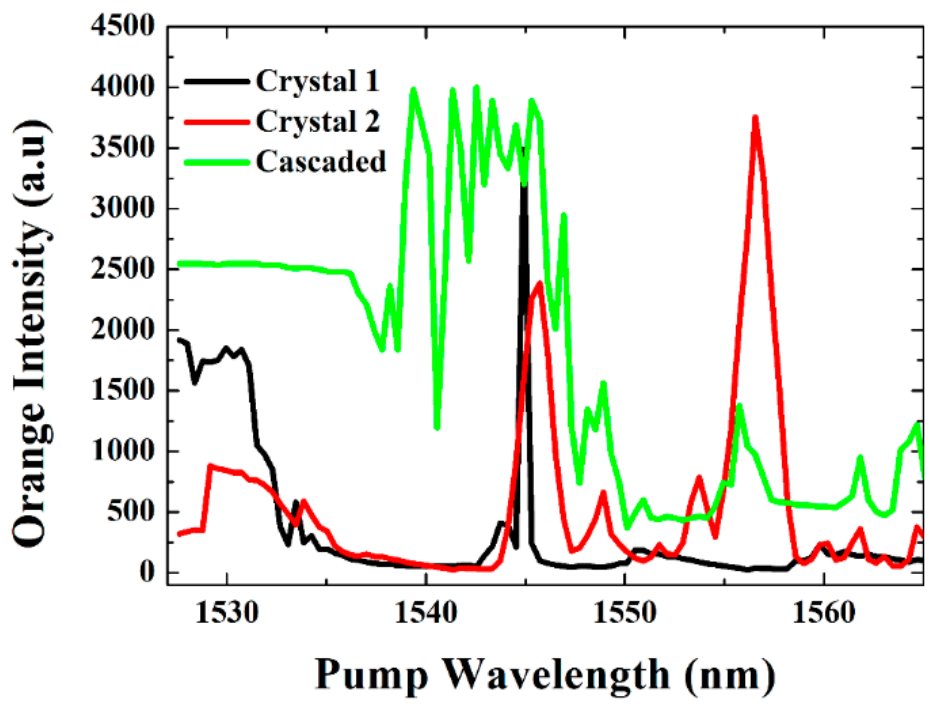

Figure 5. Measured orange laser intensity as a function of pump wavelength.

Thus, by temperature tuning, the SFG quasi phase-matching condition can be shifted owing to the fact that the refractive index of MgO:PPLN is temperature-dependent according to the Sellmeier equation. In particular, the phase-matching condition shifts to longer wavelengths with an increase in temperature. According to Figure 6, the orange light output as a function of crystal temperature can be tuned by $\sim 0.05 \mathrm{~nm} /{ }^{\circ} \mathrm{C}$ without affecting the SFG conversion efficiency for the PPLN crystal used in this work. We therefore benefit from a temperature-assisted tunable orange light source. 


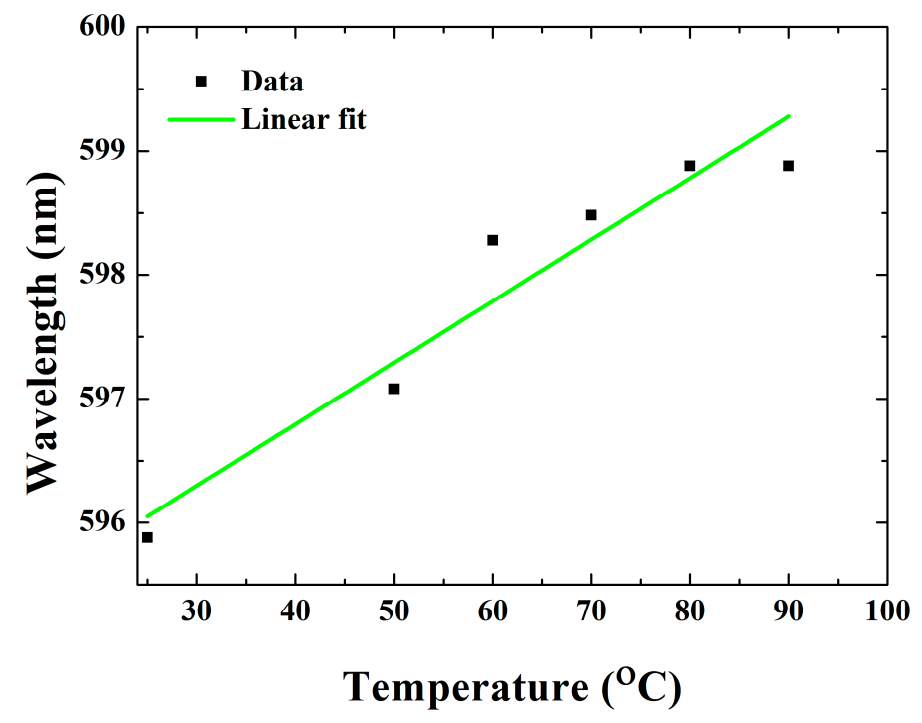

Figure 6. Variation of the generated orange light as a function of temperature tuning.

In addition, the stability of the orange light output generated by the cascaded module for one hour was measured using a power meter, showing $<2.5 \%$ fluctuation of the output power around an average of $10 \mathrm{~mW}$ (Figure 7). We attribute this fluctuation to the stability of both fundamental lasers.

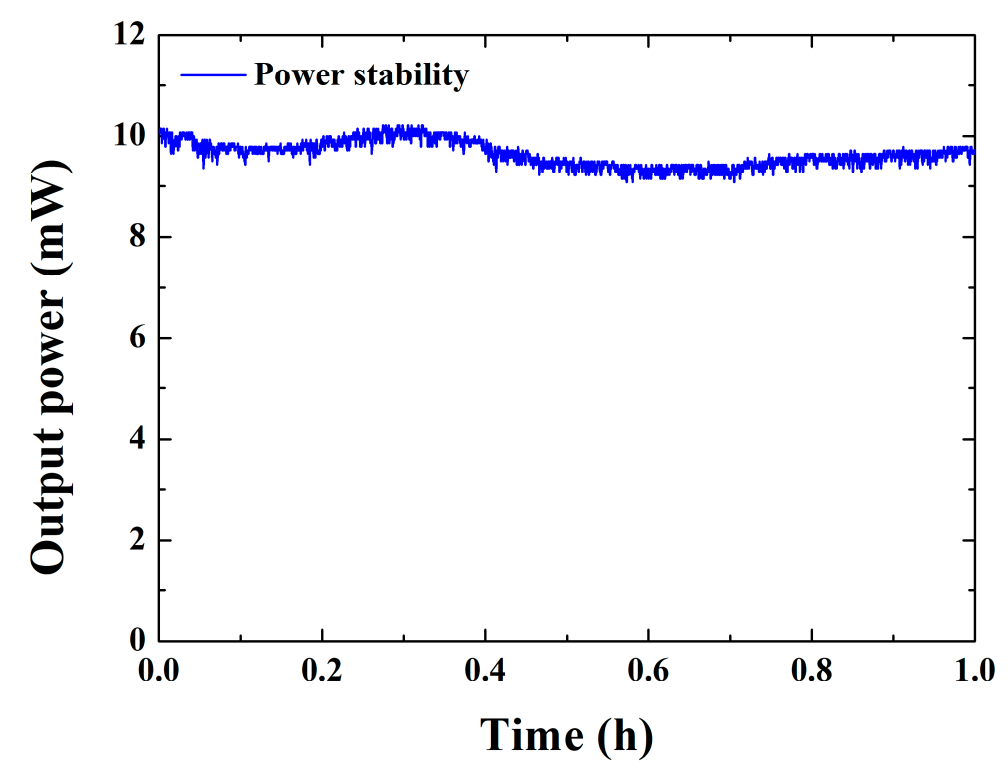

Figure 7. Stability of generated orange light with time at $596.88 \mathrm{~nm}$.

\section{Conclusions}

We have demonstrated an all-solid-state CW broadband orange laser by cascaded single-pass sum-frequency generation with fundamental wavelengths at $1545.7 \mathrm{~nm}$ and $\sim 975.1 \mathrm{~nm}$. Up to $7.1 \mathrm{~mW}$ orange output power was detected for the $50 \mathrm{~mm}$ PPLN crystal, $4.3 \mathrm{~mW}$ for the $20 \mathrm{~mm}$ SC-PPLN crystal, and $10 \mathrm{~mW}$ for the cascaded module corresponding to $7.4 \% / \mathrm{W}, 4.5 \% / \mathrm{W}$, and $10.4 \% / \mathrm{W}$ nonlinear conversion efficiencies of the total input power of the fundamental lasers, respectively. A temperature tuning rate of $\sim 0.05 \mathrm{~nm} /{ }^{\circ} \mathrm{C}$ was noted, which can be understood from the temperature dependence of the refractive index of MgO:PPLN according to the Sellmeier equation. To further improve the conversion efficiency, one will need to minimize Fresnel losses by anti-reflection coating the end faces 
of the two crystals as well as increasing the fundamental powers. We use this technique to combine the high efficiency offered by uniformly poled crystals and the broad input wavelength acceptance characteristic of step-chirped structures.

Acknowledgments: The authors gratefully acknowledge Fujian Science and Technology Service Network Initiative (STSI) Project (2016T3010).

Author Contributions: Dismas K. Choge conceived, designed, and performed the experiment; Dismas K. Choge. and Bao-Lu Tian. analyzed the experimental results; Huai-Xi Chen designed and poled the PPLN samples used in the experiment; Yi-Bin Xu and Guang-wei Li contributed in cutting, polishing, and annealing the PPLN samples; Wan-Guo Liang contributed the PPLN chips and the equipment used for preparing samples and performing experiments.

Conflicts of Interest: The authors declare no conflict of interest.

\section{References}

1. Karrer, S.; Bäumler, W.; Abels, C.; Hohenleutner, U.; Landthaler, M.; Szeimies, R.M. Long-pulse dye laser for photodynamic therapy: Investigations In Vitro and In Vivo. Lasers Surg. Med. 1999, 25, 51-59. [CrossRef]

2. Kapoor, V.; Subach, F.V.; Kozlov, V.G.; Grudinin, A.; Verkhusha, V.V.; Telford, W.G. New lasers for flow cytometry: Filling the gaps. Nat. Methods 2007, 4, 678-679. [CrossRef]

3. Marzahl, D.-T.; Metz, P.W.; Kränkel, C.; Huber, G. Spectroscopy and laser operation of Sm ${ }^{3+}$-doped lithium lutetium tetrafluoride $\left(\mathrm{LiLuF}_{4}\right)$ and strontium hexaaluminate $\left(\mathrm{SrAl}_{12} \mathrm{O}_{19}\right)$. Opt. Express 2015, 23, 21118-21127. [CrossRef] [PubMed]

4. Chang, W.K.; Chen, Y.H.; Chang, J.W. Pulsed orange generation optimized in a diode-pumped $\mathrm{Nd} \mathrm{YVO}_{4}$ laser using monolithic dual PPLN electro-optic Q switches. Opt. Lett. 2010, 35, 2687-2689. [CrossRef] [PubMed]

5. Calu, D.J.; Kawa, A.B.; Marchant, N.J.; Navarre, B.M.; Henderson, M.J.; Chen, B.; Yau, H.-J.; Bossert, J.M.; Schoenbaum, G.; Deisseroth, K.; et al. Optogenetic inhibition of dorsal medial prefrontal cortex attenuates stress-induced reinstatement of palatable food seeking in female rats. J. Neurosci. 2013, 33, $214-226$. [CrossRef] [PubMed]

6. Xu, Z.Y.; Bi, Y. Large laser projection displays utilizing all-solid-state RGB lasers. In Light-Emitting Diode Materials and Devices; SPIE Proceedings; John Wiley \& Sons Ltd.: Oboken, NJ, USA, 2005; Volume 5632, pp. 115-122.

7. Fedorova, K.A.; Cataluna, M.A.; Battle, P.R.; Kaleva, C.M.; Krestnikov, I.L.; Livshits, D.A.; Rafailov, E.U. Orange light generation from a PPKTP waveguide end pumped by a cw quantum-dot tunable laser diode. Appl. Phys. B Lasers Opt. 2011, 103, 41-43. [CrossRef]

8. Li, G.; Chen, Y.; Jiang, H.; Chen, X. Broadband sum-frequency generation using $\mathrm{d}_{33}$ in periodically poled $\mathrm{LiNbO}_{3}$ thin film in the telecommunications band. Opt. Lett. 2017, 42, 939-942. [CrossRef] [PubMed]

9. Lee, Y.L.; Noh, Y.; Jung, C.; Yu, T.J.; Ko, D.; Lee, J. Broadening of the second-harmonic phase-matching bandwidth in a temperature-gradient-controlled periodically poled $\mathrm{Ti}$ : $\mathrm{LiNbO}_{3}$ channel waveguide. Opt. Express 2003, 11, 34-35. [CrossRef]

10. Fluck, D.; Günter, P. Efficient second-harmonic generation by lens wave-guiding in $\mathrm{KNbO}_{3}$ crystals. Opt. Commun. 1998, 147, 305-308. [CrossRef]

11. Kumar, S.C.; Samanta, G.K.; Devi, K.; Fotoniques, I.D.C.; Park, M.T. High-efficiency, multicrystal, single-pass, continuous-wave second harmonic generation. Opt. Express 2011, 19, 11152-11169. [CrossRef] [PubMed]

12. Hansen, A.K.; Andersen, P.E.; Jensen, O.B.; Sumpf, B.; Erbert, G.; Petersen, P.M. Highly efficient single-pass sum frequency generation by cascaded nonlinear crystals. Opt. Lett. 2015, 40, 5526-5529. [CrossRef] [PubMed]

13. Pan, S.D.; Yu, X.Q.; Yan, Z.; Shen, Y.; Lv, X.J.; Zhu, S.N. Efficient generation of orange light in a quasi-periodically poled $\mathrm{LiTaO}_{3}$ crystal. Appl. Phys. B Lasers Opt. 2008, 93, 749-752. [CrossRef]

14. Chen, Y.L.; Chen, W.W.; Du, C.E.; Chang, W.K.; Wang, J.L.; Chung, T.Y.; Chen, Y.H. Narrow-line, cw orange light generation in a diode-pumped $\mathrm{Nd} \mathrm{YVO}_{4}$ laser using volume Bragg gratings. Opt. Express 2009, 17, 22578-22585. [CrossRef] [PubMed] 
15. Mieth, S.; Henderson, A.; Halfmann, T. Tunable, continuous-wave optical parametric oscillator with more than $1 \mathrm{~W}$ output power in the orange visible spectrum. Opt. Express 2014, 22, 11182-11191. [CrossRef] [PubMed]

16. Gayer, O.; Sacks, Z.; Galun, E.; Arie, A. Temperature and wavelength dependent refractive index equations for MgO-doped congruent and stoichiometric $\mathrm{LiNbO}_{3}$. Appl. Phys. B Lasers Opt. 2008, 91, 343-348. [CrossRef]

17. Hansen, A.K.; Tawfieq, M.; Jensen, O.B.; Andersen, P.E.; Sumpf, B.; Erbert, G.; Petersen, P.M. Concept for power scaling second harmonic generation using a cascade of nonlinear crystals. Opt. Express 2015, 23, 15921-15934. [CrossRef] [PubMed] 